\title{
A STATISTICAL HYPOTHESIS TESTING METHOD FOR THE RANK ORDERING OF THE PRIORITIES OF THE ALTERNATIVES IN THE ANALYTIC HIERARCHY PROCESS
}

\author{
Indrani Basak \\ Penn State Altoona \\ 3000 Ivyside Park \\ Altoona, PA 16601-3760 \\ E-mail: i8b@psu.edu
}

\begin{abstract}
In Analytic Hierarchy Process (AHP), a ratio scale $\left(\pi_{1}, \pi_{2}, \cdots, \pi_{t}\right)$ for the priorities of the alternatives $\left\{T_{1}, T_{2}, \cdots, T_{t}\right\}$ is used for a decision problem in which $\pi_{i} / \pi_{j}$ is used to quantify the ratio of the priority of $T_{i}$ to that of $T_{j}$. In this article, a statistical method is proposed for testing a specific rank ordering of the priorities of the alternatives.
\end{abstract}

Key Words: Hypothesis Testing, Kuhn-Tucker Problem, Likelihood-Ratio Test, Multiplicative Model, F-test.

\section{Introduction}

In the collection of the alternatives $\left\{T_{1}, T_{2}, \cdots, T_{t}\right\}$ for a decision problem, the priority vector of the alternatives is $\left(\pi_{1}, \pi_{2}, \cdots, \pi_{t}\right)$ and for each $i$ and $j$, the ratio $\pi_{i} / \pi_{j}$ gives the preference of the alternative $T_{i}$ to that of $T_{j}$. A rank ordering of the priorities of the alternatives is very important concern in a decision problem. More specifically, one needs to test a null hypothesis $H_{0}: \pi_{1}=\pi_{2}=\cdots=\pi_{t}$ against the alternative hypothesis $H_{a}: \pi_{1} \geq \pi_{2} \geq \cdots \geq \pi_{t}$ with at least one strict inequality.

Let $a_{i j}$ be the subjective estimate of $\pi_{i} / \pi_{j}$ made by a judge for some $i, j, i \neq j$. It can be assumed that $\pi_{i} / \pi_{j}$ is perturbed by the multiplicative error $e_{i j}$ to produce $a_{i j}$. Such multiplicative models has been used by Dejong (1984) and Crawford and Williams (1985). $a_{i j}$ can then be written as 


$$
a_{i j}=\frac{\pi_{i}}{\pi_{j}} e_{i j}
$$

Let $a_{i, i+1}^{(k)}$ for $k=1,2, \cdots, n$ be the estimate of $\pi_{i} / \pi_{i+1}$ provided by the $k$-th individual; $k=1,2, \cdots n .1 / a_{i+1, i}^{(k)}$ can also be taken as estimate of $\pi_{i} / \pi_{i+1}$ if $a_{i, i+1}$ estimates $\pi_{i} / \pi_{i+1}$. Other values of $a_{i j}$ for $i \neq j$ can also be multiplied to produce estimates of $\pi_{i} / \pi_{i+1}$. In this situation, it is appropriate that the common distribution of $a_{i j}$ to be reciprocal symmetric (see Crawford and Williams (1985)). For the multiplicative model (1), one such distribution is the log-normal distribution. We assume here that $e_{i j} s$ are independent and have lognormal distribution with mean zero and variance $\sigma^{2}$. Basak (1990) proposed a statistical hypothesis testing method to test the null hypothesis $H_{0}$ against the alternative hypothesis $H_{a}$. But the corresponding distribution of the test criterion was rather complicated. In Section 2, we propose simple alternatives of the null hypothesis $H_{0}$ and the alternative hypothesis $H_{a}$ along with its test criterion. Computation of the proposed test criterion and its distribution is discussed further in Section 3. A numerical example is provided in Section 4 to illustrate the methodology proposed in this article. We then make some concluding remarks in Section 5.

\section{The Hypothesis Testing Problem}

In the selection problem, the decision making is heavily dependent on the ranking of the alternatives. The relative standing of the priority alternatives is important in resource allocation problems too. In those problems, ranking of the alternatives would be sufficient for the selection of a number of highly preferred alternatives. In these cases, one would like to assure that the ranking of the alternatives is well-supported by the statistical theory.

A problem of hypothesis testing is proposed in this section. The hypothesis which gives a specific ranking of the alternatives, without loss of generality, is the alternative hypothesis $H_{a}: \pi_{1} \geq \pi_{2} \geq \cdots \geq \pi_{t}$ in favor of which we want to get evidence using sample information. At least one is a strict inequality in the string of inequalities in $H_{a}$. The hypothesis we want to reject is called the null hypothesis, $H_{0}$. It states that all the alternatives are of equal standing, i.e., $H_{0}: \pi_{1}=\pi_{2}=\cdots=\pi_{t}$.

Therefore, one has to test

$$
\left.\begin{array}{l}
H_{0}: \pi_{1}=\pi_{2}=\cdots=\pi_{t} \text { against } \\
H_{a}: \pi_{1} \geq \pi_{2} \geq \cdots \geq \pi_{t} \text { with at least one strict ineqiality. }
\end{array}\right\}
$$

The hypothesis testing problem in (2) has the following equivalent form:

$$
\left.\begin{array}{r}
H_{0}: \theta_{i}=\ln \pi_{i}-\ln \pi_{i+1}=0 \text { for } i=1, \cdots, t-1 \text { against } \\
H_{a}: \theta_{i}=\ln \pi_{i}-\ln \pi_{i+1} \geq 0 \text { for } i=1, \cdots, t-1 \\
\quad \text { with at least one strict inequality. }
\end{array}\right\}
$$


The likelihood function $f$ of $a_{i, i+1}^{(k)}$ for $i=1,2, \cdots, t-1$ and $k=1,2, \cdots, n$ is proportional to

$$
\exp \left[-\frac{1}{2 \sigma^{2}} \sum_{i=1}^{t-1}\left\{\sum_{k=1}^{n}\left(\ln a_{i, i+1}^{(k)}-\overline{\ln } a_{i, i+1}\right)^{2}+n\left(\ln a_{i, i+1}+\ln \pi_{i+1}-\ln \pi_{i}\right)^{2}\right\}\right]
$$

in which $\overline{\ln } a_{i, i+1}=\frac{1}{n} \sum_{k=1}^{n} \ln a_{i, i+1}^{(k)}$. Writing $\theta_{i}=\ln \pi_{i}-\ln \pi_{i+1}$ and $y_{i}=\overline{\ln } a_{i, i+1}$ in the equation (4), one gets the likelihood function $f$ to be proprtional to

$$
\exp \left[-\frac{n}{2 \sigma^{2}} \sum_{i=1}^{t-1}\left(y_{i}-\theta_{i}\right)^{2}\right]
$$

To test the null hypothesis $H_{0}$ against the alternative hypothesis $H_{a}$, each of which are given by (3), we will use the well-known statistical method of the likelihood ratio test. The likelihood ratio test is based on whether

$$
\begin{gathered}
\lambda=\max _{\Theta_{0}} f / \max _{\Theta_{a}} \text { is small or not, or equivalently, } \\
\lambda^{*}=-2 \ln \lambda=2\left[\min _{\Theta_{0}}(-\ln f)-\min _{\Theta_{a}}(-\ln f)\right] \quad \text { is large or not. }
\end{gathered}
$$

In the above, denoting $m=t-1$,

$$
\begin{aligned}
\Theta_{0} & =\left\{\theta_{i} \mid \theta_{i}=0, i=1,2, \cdots, m\right\} \\
\Theta_{a} & =\left\{\theta_{i} \mid \theta_{i} \geq 0, i=1,2, \cdots, m \text { with at least one strict inequality }\right\}
\end{aligned}
$$

By using (5), $\lambda^{*}$ in (6) can easily be evaluated to be

$$
\left.\lambda^{*}=n / s^{2}\left[\sum_{i=1}^{m} y_{i}^{2}-\min _{\Theta_{a}} \sum_{i=1}^{m}\left(y_{i}-\theta_{i}\right)^{2}\right]\right)
$$

where $s^{2}$ is an estimate of $\sigma^{2}$.

\section{Computation of the test criterion $\lambda^{*}$ and its distribution under $H_{0}$}

The minimum of the convex function $f^{*}=n \sum_{i=1}^{m}\left(y_{i}-\theta_{i}\right)^{2}$ of $\theta_{i}$ has to be found under the inequality constraints $g_{i}\left(\theta_{1}, \theta_{2}, \cdots, \theta_{m}\right)=\theta_{i} \geq 0[$ for $i=1,2, \cdots, m]$ in order to compute the test criterion $\lambda^{*}$. This problem can be readily seen to be a non-linear programming (NLP) problem. The NLP problem can be stated as follows: 
Find $\theta_{i}, u_{i}, i=1,2, \ldots, m$, that satisfy

$$
\begin{aligned}
\frac{\partial f^{*}}{\partial \theta_{i}} & =\sum_{k=1}^{m} u_{k} \frac{\partial g_{k}}{\partial \theta_{i}} \\
g_{i}\left(\theta_{1}, \theta_{2}, \cdots, \theta_{m}\right) & \geq 0 \\
u_{i} & \geq 0 \\
u_{i} . g_{i}\left(\theta_{1}, \theta_{2}, \cdots, \theta_{m}\right) & =0
\end{aligned}
$$

Assuming that the functions $f^{*}$ and $g$ are differentiable, we state the necessary and sufficient optimality conditions for this NLP problem. These optimality conditions are commonly known as the Kuhn-Tucker conditions. Solving for these Kuhn-Tucker conditions is also referred as Kuhn-Tucker (KT) problem. Solving for KT problem is finding a solution to a system of non-linear equations (8). We state below necessary and sufficient optimality conditions for the KT problem.

Theorem 1 (Kuhn-Tucker Necessity Theorem): If the objective function $f^{*}$ and the constraint functions $g_{i}$ are differentiable and if, furthermore $\partial g_{i}\left(\theta_{1}, \cdots, \theta_{m}\right) / \partial \theta_{i}$ for $i=1,2, \cdots, m$ are linearly independent (this property is called the constraint qualification), then if $\left(\theta_{1}^{*}, \cdots, \theta_{m}^{*}\right)$ is an optimal solution to the NLP problem, then there exist $\left(u_{1}^{*}, \cdots, u_{m}^{*}\right)$ such that $\left(\theta_{1}^{*}, \cdots, \theta_{m}^{*}, u_{1}^{*}, \cdots, u_{m}^{*}\right)$ solve the KT problem.

It is to be noted that $f^{*}$ and $g_{i}$ for $i=1,2, \cdots, m$ are differentiable functions in this situation and the constraint qualification is also satisfied.

Theorem 2 (Kuhn-Tucker Sufficiency Theorem): If the objective function $f^{*}$ is convex and the inequality constraints $g_{i}$ are all concave functions and if there exists a solution $\left(\theta_{1}^{*}, \cdots, \theta_{m}^{*}, u_{1}^{*}, \cdots, u_{m}^{*}\right)$ that satisfies the $\mathrm{KT}$ problem, then $\left(\theta_{1}^{*}, \cdots, \theta_{m}^{*}\right)$ is an optimal solution to the NLP problem.

It is to be noted that our objective function $f^{*}$ is convex and our inequality constraints are concave.

Therefore, the existence of the optimal solutions $\left(\theta_{1}^{*}, \cdots, \theta_{m}^{*}\right)$ are guaranteed by the above two theorems. We will now proceed to obtain these $\theta_{1}^{*}, \cdots, \theta_{m}^{*}$ explicitly. The gradient vectors of $g_{i}$ for $i=1,2, \cdots, m$ are $\frac{\partial g_{i}}{\partial \theta_{i}}=(0,0, \cdots, 1,0, \cdots, 0)$ in which 1 occurs in the $i$-th position. The equations given by $(8)$ reduce to

$$
\left.\begin{array}{rl}
2 n\left(\theta_{i}-y_{i}\right) & =u_{i}, \\
\theta_{i} & \geq 0, \\
u_{i} & \geq 0 \\
u_{i} \cdot \theta_{i} & =0 .
\end{array}\right\}
$$


One gets the following by solving (9) for $\theta_{i}$ and $u_{i}$ for $i=1,2, \cdots, m$ :

Either $u_{i}=0$ in which case $\theta_{i}=y_{i}$ or $u_{i}=-2 n y_{i}$ in which case $\theta_{i}=0$.

Now, let $R$ denote the space of all sample vectors $\left(y_{1}, y_{2}, \cdots, y_{m}\right)$. A point $\left(y_{1}^{0}, y_{2}^{0}, \cdots, y_{m}^{0}\right)$ is known as the maximum likelihood estimate of $\left(\theta_{1}, \theta_{2}, \cdots, \theta_{m}\right)$ for which the function $f^{*}=n \sum_{i=1}^{m}\left(y_{i}-\theta_{i}\right)^{2}$ is minimized. More specifically,

$$
\operatorname{minf} f^{*}=n \sum_{i=1}^{m}\left(y_{i}-y_{i}^{0}\right)^{2}
$$

Next we develop a testing procedure following Bartholomew (1959a, 1959b) and Kudo (1963) in which we find the maximum likelihood estimate $\left(y_{1}^{0}, y_{2}^{0}, \cdots, y_{m}^{0}\right)$. Unlike Bartholomew (1959a, 1959b) and Kudo (1963) who used an heuristic algorithm, we find the maximum likelihood estimate using non-linear programming technique. For that purpose, the space of all sample vectors $R$ can be thought of divided into $2^{m}$ disoint subsets,

$$
R=U_{\phi \subset M \subset A} R_{M},
$$

in which $R_{M}$ is the totality of all points $\left(y_{1}, y_{2}, \cdots, y_{m}\right)$ such that $y_{i}^{0}=0$ for $i \notin M$ and $y_{i}^{0}>0$ for $i \in M$ where $\left(y_{1}^{0}, y_{2}^{0}, \cdots, y_{m}^{0}\right)$ are the maximum likelihood estimates associated with $\left(y_{1}, y_{2}, \cdots, y_{m}\right)$. In (11), $A=\{1,2, \cdots, m\}$ and $\phi$ is the null set. Clearly, $R_{A}$ is the totality of all the sample vectors for which every component of the maximum likelihood vector is greater than zero and $R_{\phi}$ is the totality of all the sample vectors for which every component of the maximum likelihood vector is zero. It is to be recalled now that the existence of the optimal solutions $\left(\theta_{1}^{*}, \cdots, \theta_{m}^{*}\right)$ are guaranteed by Theorems 1 and 2. These optimum solutions $\left(\theta_{1}^{*}, \cdots, \theta_{m}^{*}\right)$ are actually the maximum likelihood estimates $\left(y_{1}^{0}, y_{2}^{0}, \cdots, y_{m}^{0}\right)$. Therefore, the existence of the maximum likelihood estimates $\left(y_{1}^{0}, y_{2}^{0}, \cdots, y_{m}^{0}\right)$ are guaranteed for any sample vector $\left(y_{1}, y_{2}, \cdots, y_{m}\right)$. In the following theorem, we will provide a necessary and sufficient condition for a sample vector $\left(y_{1}, y_{2}, \cdots, y_{m}\right)$ to belong to $R_{M}$. We will denote $M=\{d+1, \cdots, m\}$ in order to avoid confusing notations but it will be clear that the method works for any $M$ for $\phi \subset M \subset A$.

Theorem 3: A necessary and sufficient condition for the sample vector $\left(y_{1}, y_{2}, \cdots, y_{m}\right)$ belonging to $R_{M}$ where $\phi \subset M \subset A$ and $M=\{d+1, \cdots, m\}$ is

(i) $y_{i} \leq 0$ for $i=1,2, \cdots, d$,

(ii) $y_{i}>0$ for $i=d+1, d+2, \cdots, m$.

Proof: (Necessity) If the sample vector $y$ belongs to $R_{M}$ then $y_{i}^{0}>0$ for $i=d+1, \cdots, m$. Since in this case $y_{i}^{0}=y_{i}$ using (10), we have $y_{i}>0$ for $i=d+1, d+2, \cdots, m$. This proves (ii). To prove (i), let us assume the contrary. It means $y_{i}>0$ for some $i=1,2, \cdots, d$. Then for that $i$, using (10), we get $y_{i}^{0}>0$ which is contradictory to our assumption that the 
sample vector $\left(y_{1}, y_{2}, \cdots, y_{m}\right)$ belongs to $R_{M}$. Hence (i) is proved and so is the necessity part of the theorem.

(Sufficiency) For this part, we have to show that (i) and (ii) imply that the sample vector $\left(y_{1}, y_{2}, \cdots, y_{m}\right)$ belongs to $R_{M}$. It means that we need to show that $y_{i}^{0}>0$ for $i=$ $d+1, \cdots, m$ and $y_{i}^{0}=0$ for $i=1,2, \cdots, d$. (ii) and (10) together imply that $y_{i}^{0}=y_{i}>0$ for $i=d+1, \cdots, m$. Now, if $y_{i}<0$ in (i) for some $i=1,2, \cdots, d$, then using $\left(10, u_{i}>0\right.$ for that $i$. It follows, then, from the fourth equation of (9) that $y_{i}^{0}=0$ for that $i$. Also, if $y_{i}=0$ for some $i=1,2, \cdots, d$ then we get, from (10), that $y_{i}^{0}=y_{i}=0$ for that $i$. In any case, $y_{i}^{0}=0$ for $i=1,2, \cdots, d$. This proves the sufficiency part of the theorem. Hence Theorem 3 is proved. In light of Theorem 3 and (10), $\lambda^{*}$ in (7) then reduces to

$$
\lambda^{*}=\frac{n}{s^{2}} \sum_{i \in m} y_{i}^{2} .
$$

In the following theorem, we provide the distribution of $\lambda^{*}$ given by (12) under the null hypothesis $H_{0}$ given in (3).

Theorem 4: Under the null hypothesis $H_{0}: \theta_{i}=0$ for $i=1,2, \cdots, m$, $\frac{n m-1}{m-d} \lambda^{*}=$ $\frac{n(n m-1)}{(m-d) s^{2}} \sum_{i \in M} y_{i}^{2}$ has $F$ distribution with the numerator degrees of freedom $(m-d)$ and the denominator degrees of freedom $(n m-1)$ where $s^{2}=\sum_{k=1}^{n} \sum_{i=1}^{m}\left[\ln a_{i, i+1}^{(k)}-\bar{a}\right]^{2}$ with $\bar{a}=$ $\sum_{k=1}^{n} \sum_{i=1}^{m} \ln a_{i, i+1}^{(k)} /(n \cdot m)$

Proof: Since $\ln a_{i, i+1}^{(k)}$ is normally distributed with mean $\theta_{i}$ and variance $\sigma^{2}, y_{i}$ has mean $\theta_{i}$ and variance $\sigma^{2} / n$. Therefore, under the null hypothesis $H_{0}, \sqrt{n} y_{i}$ has mean 0 and variance $\sigma^{2}$ for $i=d+1, \cdots, m$. Consequently, $n \sum_{i \in M} y_{i}^{2}$, under $H_{0}$, is distributed as $\sigma^{2} \cdot \chi_{m-d}^{2}$ where $\chi_{m-d}^{2}$ represents chi-square distribution with $(m-d)$ degrees of freedom.

Now, under $H_{0}, s^{2}=\sum_{k=1}^{n} \sum_{i=1}^{m}\left[\ln a_{i, i+1}^{(k)}-\bar{a}\right]^{2}$, [where $\left.\bar{a}=\sum_{k=1}^{n} \sum_{i=1}^{m} \ln a_{i, i+1}^{(k)} /(n \cdot m)\right]$ is distributed as $\sigma^{2} \cdot \chi_{n m-1}^{2}$ where $\chi_{n m-1}^{2}$ represents chi-square distribution with $(n m-1)$ degrees of freedom. Moreover, $n \sum_{i \in M} y_{i}^{2}$ is distributed independently of $s^{2}$ under $H_{0}$. Therefore, under $H_{0}$

$$
\frac{n \sum_{i \in M} y_{i}^{2} / m-d}{s^{2} / n m-1} \sim F_{m-d, n m-1}
$$

in which $F_{m-d, n m-1}$ represents $F$ distribution with numerator degrees of freedom $(m-d)$ and denominator degrees of freedom $(n m-1)$. This, in turn, proves that $\frac{n m-1}{m-d} \lambda^{*}$ has $F$ 
distribution with the numerator degrees of freedom $(m-d)$ and the denominator degrees of freedom $(n m-1)$.

The above distribution of $\frac{n m-1}{m-d} \lambda^{*}$ can be used to test the hypothesis in (2). At significance level $\alpha$, one would reject $H_{0}$ if

$$
\frac{n m-1}{m-d} \lambda^{*} \geq F_{0}
$$

where $F_{0}$ is the corresponding critical value of $F_{m-d, n m-1}$ distribution, i.e, $P\left[F_{m-d, n m-1} \geq\right.$ $\left.F_{0}\right]=\alpha$. In the next section, we provide an numerical example to illustrate the testing method we proposed in this section.

\section{Numerical Illustration}

In order to illustrate the testing method developed in the paper, we present an artificial data on the school selection example given by (Saaty, 1980) in this section. In an hypothetical experiment, seven independent observations on each of the thirty pairs of six criteria [Learning (L), Friends (F), School Life (S), Vocational Training (V), College Preparatiopn (C) and Music Classes (M)] were collected from seven individuals of similar background. The data are provided in Table 1. For example, the fifth observation on the pair $(\mathrm{L}, \mathrm{F})$ is 2.5 means that $\mathrm{L}$ is preferred 2.5 times $\mathrm{F}$ by the fifth individual.

These data were used by Basak (2011) and using the method provided in that article, the rank order of the priorities of six criteria was established as $\pi_{L}>\pi_{C}>\pi_{F}=\pi_{V}=\pi_{M}=\pi_{S}$. We wanted to check whether the methodology proposed in this article would support the same ranking. In other words, we would like to test $H_{0}: \pi_{L}=\pi_{C}=\pi_{F}=\pi_{V}=\pi_{M}=\pi_{S}$ vs $H_{a}: \pi_{L} \geq \pi_{C} \geq \pi_{F} \geq \pi_{V} \geq \pi_{M} \geq \pi_{S}$ with at least one strict inequality.

The test criterion $\frac{n m-1}{m-d} \lambda^{*}$ is computed to be 5.524. Recall that, Under $H_{0}, \frac{n m-1}{m-d} \lambda^{*}$ is distributed as $F_{m-d, n m-1}$ distribution. Here, $(m-d)=4$ and $n m-1=34$. At $1 \%$ 
Table 1. Comparisons Data of six criteria with respect to overall satisfaction with school; $\mathbf{n}=7$.

\begin{tabular}{|c|ccccccc|}
\hline & \multicolumn{7}{|c|}{ Observations } \\
\cline { 2 - 8 } Pairs & 1 & 2 & 3 & 4 & 5 & 6 & 7 \\
\hline (L,F) & 4 & 3 & 2 & 1.5 & 2.5 & 4 & 3.5 \\
(L,S) & 3 & 2.5 & 2 & 3.2 & 2 & 2.5 & 3 \\
(L,V) & 1 & 1.5 & 0.5 & 1 & 0.5 & 2 & 1 \\
(L,C) & 3 & 2.5 & 4 & 3.5 & 3 & 2 & 2.5 \\
(L,M $)$ & 4 & 3 & 3.5 & 3 & 4 & 3 & 3.5 \\
(F,L) & 0.2 & 0.25 & 0.5 & 0.5 & 0.67 & 0.33 & 0.25 \\
(F,S) & 6 & 3.5 & 4 & 4.5 & 5 & 7 & 6.5 \\
(F,V) & 3 & 2.5 & 3 & 4 & 3.5 & 2 & 2.5 \\
(F,C) & 0.2 & 0.25 & 0.33 & 0.2 & 0.17 & 0.2 & 0.25 \\
(F,M $)$ & 1 & 1.5 & 1 & 2 & 1.5 & 0.5 & 2 \\
(S,L) & 0.5 & 0.33 & 0.5 & 0.33 & 0.33 & 0.5 & 0.33 \\
(S,F) & 0.2 & 0.25 & 0.33 & 0.2 & 0.17 & 0.17 & 0.17 \\
(S,V) & 0.2 & 0.25 & 0.2 & 0.25 & 0.33 & 0.2 & 0.25 \\
(S,C) & 0.17 & 0.25 & 0.2 & 0.33 & 0.5 & 0.2 & 0.33 \\
(S,M) & 0.2 & 0.25 & 0.33 & 0.25 & 0.25 & 0.2 & 0.33 \\
(V,L) & 0.5 & 0.5 & 3 & 0.5 & 2 & 0.5 & 0.5 \\
(V,F) & 0.5 & 0.33 & 0.5 & 0.2 & 0.25 & 0.5 & 0.33 \\
(V,S) & 4 & 3 & 4 & 4 & 3 & 4 & 3 \\
(V,C) & 1 & 0.5 & 2 & 0.5 & 1 & 1.5 & 2 \\
(V,M) & 0.33 & 0.5 & 0.5 & 0.33 & 0.5 & 1 & 0.5 \\
(C,L) & 0.5 & 0.33 & 0.25 & 0.33 & 0.5 & 0.33 & 0.5 \\
(C,F) & 4 & 3 & 2 & 4 & 5 & 5 & 3 \\
(C,S) & 5 & 3 & 5 & 2 & 3 & 4 & 3 \\
(C,V) & 1 & 2 & 0.5 & 3 & 0.5 & 0.5 & 0.33 \\
(C,M) & 3 & 2 & 2.5 & 3.5 & 3 & 2 & 3.5 \\
(M,L) & 0.2 & 0.5 & 0.25 & 0.25 & 0.25 & 0.5 & 0.33 \\
(M,F) & 1 & 0.5 & 0.5 & 0.33 & 0.5 & 3 & 0.33 \\
(M,S) & 4 & 3 & 4 & 3 & 5 & 4 & 3 \\
(M,V) & 4 & 3 & 2 & 4 & 3 & 1 & 3 \\
(M,C) & 0.33 & 0.33 & 0.5 & 0.25 & 0.33 & 0.5 & 0.25 \\
\hline & & & & & & & \\
\hline
\end{tabular}

significance level, the critical value is $F_{0}=3.927$. Therefore, we reject $H_{0}$ at $1 \%$ significance level and hence the rank order of the priorities $\pi_{L}>\pi_{C}>\pi_{F}=\pi_{V}=\pi_{M}=\pi_{S}$ is supported by the methodology proposed in this article too. One advantage of the methodology of this article is that one does not need all of the above thirty pairwise comparisons data. Only $(\mathrm{L}, \mathrm{C}),(\mathrm{C}, \mathrm{F}),(\mathrm{F}, \mathrm{V}),(\mathrm{V}, \mathrm{M})$ and $(\mathrm{M}, \mathrm{S})$ are sufficient to obtain from different individuals. Therefore, one would only need the following Table 2. 
Table 2. Comparisons Data needed for the proposed testing method; $\mathbf{n}=7$.

\begin{tabular}{|c|ccccccc|}
\hline & \multicolumn{7}{|c|}{ Observations } \\
\cline { 2 - 8 } Pairs & 1 & 2 & 3 & 4 & 5 & 6 & 7 \\
\hline (L,C) & 3 & 2.5 & 4 & 3.5 & 3 & 2 & 2.5 \\
(C,F) & 4 & 3 & 2 & 4 & 5 & 5 & 3 \\
$(\mathrm{~F}, \mathrm{~V})$ & 3 & 2.5 & 3 & 4 & 3.5 & 2 & 2.5 \\
$(\mathrm{~V}, \mathrm{M})$ & 0.33 & 0.5 & 0.5 & 0.33 & 0.5 & 1 & 0.5 \\
$(\mathrm{M}, \mathrm{S})$ & 4 & 3 & 4 & 3 & 5 & 4 & 3 \\
\hline
\end{tabular}

Next, we would be testing $H_{0}: \pi_{S}=\pi_{M}=\pi_{V}=\pi_{F}=\pi_{C}=\pi_{L}$ vs $H_{a}: \pi_{S} \geq \pi_{M} \geq \pi_{V} \geq$ $\pi_{F} \geq \pi_{C} \geq \pi_{L}$ with at least one strict inequality. We would then only need the following Table 3 .

Table 3. Comparisons Data needed for the proposed testing method; $\mathbf{n}=7$.

\begin{tabular}{|c|ccccccc|}
\hline & \multicolumn{7}{|c|}{ Observations } \\
\cline { 2 - 8 } Pairs & 1 & 2 & 3 & 4 & 5 & 6 & 7 \\
\hline$(\mathrm{S}, \mathrm{M})$ & 0.2 & 0.25 & 0.33 & 0.25 & 0.25 & 0.2 & 0.33 \\
$(\mathrm{M}, \mathrm{V})$ & 4 & 3 & 2 & 4 & 3 & 1 & 3 \\
$(\mathrm{~V}, \mathrm{~F})$ & 0.5 & 0.33 & 0.5 & 0.2 & 0.25 & 0.5 & 0.33 \\
$(\mathrm{~F}, \mathrm{C})$ & 0.2 & 0.25 & 0.33 & 0.2 & 0.17 & 0.2 & 0.25 \\
$(\mathrm{C}, \mathrm{L})$ & 0.5 & 0.33 & 0.25 & 0.33 & 0.5 & 0.33 & 0.5 \\
\hline
\end{tabular}

The test criterion $\frac{n m-1}{m-d} \lambda^{*}=n / s^{2} \sum_{i \in M} y_{i}^{2}$ in this case was computed to be 5.392. Here, $(m-d)=1$ and $(n m-1)=34$. At $1 \%$ significance level, the critical value is $F_{0}=7.444$. Therefore, we would not reject $H_{0}$ at $1 \%$ significance level and hence the rank order of the priorities $\pi_{S} \geq \pi_{M} \geq \pi_{V} \geq \pi_{F} \geq \pi_{C} \geq \pi_{L}$ with at least one strict inequality is not supported.

\section{Concluding Remark}

In the present article, we proposed a simple statistical method for testing rank ordering of the priorities of the alternatives in the Analytic Hierarchy Process. It is found that this proposed method is much simpler compared with the other methods but produces similar conclusion.

\section{REFERENCES}

Aczel, J. and Saaty, T. L. (1983). Procedures for synthesizing ratio judgments. Journal of Mathematical Psychology, 27, 93-102.

Bartholomew, D. J. (1959). A test of homogeneity for ordered alternatives. Biometrika, 46, 36-48. 
Bartholomew, D. J. (1959). A test of homogeneity for ordered alternatives. II. Biometrika, 46, 328- 335 .

Basak, I. (1990). Testing for the rank ordering of the priorities of the alternatives in Saaty's ratio-scale method. European Journal of Operational Research, 48, 148-152.

Basak, I. (2011). An alternate method of deriving priorities and related inferences for group decision making in Analytic Hierarchy Process. Journal of Multi-Critetria Decision Analysis, 18, 279-287.

Crawford, G. and Williams, C. (1985). A note on analysis of subjective judgment matrices. Journal of Mathematical Psychology, 29, 387-405.

Dejong, P. (1984). A statistical approach to Saaty's scaling method for priorities. Journal of Mathematical Psychology, 28, 467-478.

Kudo, A. (1963). A multivariate analogue of the one-sided test. Biometrika, 50, 403-418.

Saaty, T. L. (1966). A scaling method for priorities in hierarchical structures. Journal of Mathematical Psychology, 5, 234-281.

Saaty, T.L. (1980). The Analytic Hierarchy Process, McGraw-Hill.

Vargas, L.G. (1982). Reciprocal matrices with random coefficients. Mathematical Modelling, 3, 69-81.

Zahedi, F. (1986). The Analytic Hierarchy Process - a survey of the method and its applications. Interfaces, 16, 96-108. 